\title{
PacMan for Biotechnology: Co-opting Degrons for Targeted Protein Degradation to Control and Alter Cell Function
}

\author{
Geng Yu ${ }^{\mathrm{a}}$, Julian N. Rosenberg ${ }^{\mathrm{a}}$, Michael J. Betenbaugh ${ }^{\mathrm{a}}$, George A. Oyler ${ }^{\mathrm{a}, \mathrm{b}}$ \\ ${ }^{a}$ Department of Chemical and Biomolecular Engineering, Johns Hopkins University, Baltimore, \\ MD, USA 21218

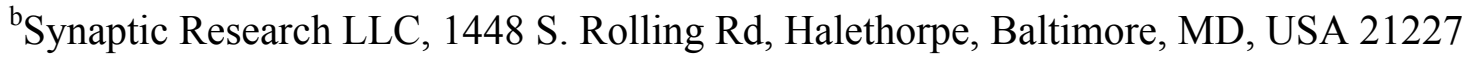

Corresponding Author: $\quad$ George A. Oyler, george@synapticresearch.com

$+01-443-543-5906$ 


\begin{abstract}
Protein degradation in normal living cells is precisely regulated to match the cells' physiological requirements. The selectivity of protein degradation is determined by an elaborate degrontagging system. Degron refers to an amino acid sequence that encodes a protein degradation signal, which is oftentimes a poly-ubiquitin chain that can be transferred to other proteins. Current understanding of ubiquitination dependent and independent protein degradation processes has expanded the application of degrons for targeted protein degradation and novel strategy development. Recent findings suggest that small molecules induced protein association can be exploited to create degrons in targeted protein degradations. Here, recent applications of degron-based targeted protein degradation in eukaryotic organisms are reviewed. The degron mediated protein degradation represents a rapidly tunable methodology to control protein abundance, which has broadened its application in therapeutics and cellular function control and monitoring.
\end{abstract}




\section{Introduction}

Protein degradation in living cells is a highly selective process in which damaged or misfolded proteins are readily targeted for destruction. However, this process extends far beyond the removal of inactive proteins. The relative abundance of hundreds of regulatory proteins is modulated by degradative processes in concert with the cell growth-and-division cycle as well as diurnal rhythms $\left[1^{\bullet}\right]$. The targeting specificity of protein degradation machinery is determined by "degrons" that are attached to or carried by the targeted protein. The term "degron" was first proposed by Varshavsky [2], referring to an amino acid sequence that serves as a destruction signal for proteins whose stability follows the $\mathrm{N}$-end rule, which relates the in vivo stability of a protein to the identity of its N-terminal residue [3]. Degron is now used as a general term for all transferrable degradation signals that can be attached to a protein of interest (POI) for targeted protein degradation (TPD). "N-degron" is reserved for transferrable N-terminal amino acids that will cause the target protein degradation following the $\mathrm{N}$-end rule [3], which relates the in vivo stability of a protein to the identity of its $\mathrm{N}$-terminal residue. The unstable nature of the $\mathrm{N}$-degron is attributed to its first amino acids, which are prone to acetylation or arginylation modifications and ultimately lead to ubiquitination and degradation.

A degron requires at least two components to ensure TPD: (1) a target protein degradation recognition tag, such as a poly-ubiquitin tag, and (2) an unstructured region in close proximity to the tag. Both elements are required for protein degradation. For example, the ubiquitinated yeast $\operatorname{Rad} 23$ protein can evade proteasome degradation due to lack of an unstructured region $[4,5]$. However, the recognition tag and unstructured region are not required to be present in cis on the 
targets. For instance, a multi-peptide complex can be degraded as a whole with one peptide serving as the ubiquitination site and another peptide providing the unstructured region [6].

Since protein degradation in a cell is mainly carried out by the proteasome in eukaryotic organisms, with a small portion by cytosolic or nuclear proteases and lysosomal or autophagic proteolysis, only eukaryotic proteasome-mediated TPD will be discussed in this review. Prokaryotic protease mediated protein degradation is reveiewd in [7]. The 26S proteasome is composed of the cylindrical 20S core particle and regulatory particles at both ends of the core (Figure 1). The $20 \mathrm{~S}$ core consists of four stacked heptameric rings, namely $\alpha-\beta-\beta-\alpha$ rings, to form a cavity. The 19S complex caps on the ends of the cavity to mediate the substrates recognition, unfolding and transporting. Degron-mediated protein degradation in the proteasome can be roughly classified into ubiquitination-based or ubiquitination-independent pathways [8]. Ubiquitination-mediated protein degradation is the most common degron system and has been extensively reviewed $[9,10]$. In this process, a cascade of E1, E2 and E3 enzymes activate the ubiquitin monomer and attach it to the targeted substrates in order to initiate poly-ubiquitin chain formation (Figure 1). These poly-ubiquitin chains consist of intermolecular Lys-48 linkages and are typically attached to multiple residues on the substrate. De-ubiquitination enzymes (DUB) also function in the cell to reverse the poly-ubiquitin chain growth, adding a check-point for target selection [11]. The protein destruction-recognition signal is encrypted by the length, number, position and linkage method of these poly-ubiquitin chains. The delivery of the polyubiquitinated proteins to the proteasome is mediated by the interaction between the polyubiquitin tag and the receptors on the $19 \mathrm{~S}$ complex of the proteasome [12]. N-degrons can be viewed as special transferrable $\mathrm{N}$-terminal peptides encrypting the ubiquitination initiation signal. 
The degron system for ubiquitin-independent protein degradation has been elucidated principally for the C-degron on ODC (Ornithine decarboxylase) [13]. In this process, ODC degradation is mediated by antizyme 1 (AZ1) bridged association of ODC to the proteasome through a physical interaction with a 36 amino-acid sequence from the carboxyl-terminal of ODC (Figure 1). Since this 36 amino-acid sequence from ODC can be attached to the carboxyl-terminus of numerous target proteins to cause their degradation, it is termed a "C-degron". Thus, the AZ1 bound Cdegron serves as both the recognition and degradation initiation signal for the proteasome in an ubiquitin-independent pathway.

All of the aforementioned types of protein targeting and degradation methods known in nature have been engineered in the form of degrons to a large variety of POI for TPD [14,15]. This review will provide a brief overview on the engineering of degrons in the forms of ubiquitination dependent and independent protein degradation pathways together with recent TPD applications utilizing the degron concept derived from these pathways.

\section{Ubiquitination-dependent protein degradation pathway for TPD}

Analogous to the naturally occurring ubiquitination dependent protein degradation pathway, the early attempt to engineer targeted protein degradation was achieved by engineering E3 ligases (Figure 2A). Since E3 ligases are structurally flexible and can accommodate substrates with different sizes and shapes, changing the substrate recognition domain of E3 ligases will allow selective ubiquitination and degradation of POI. The first in vivo example was the exploitation of the interaction between the human papillomavirus protein E7 (E7N) and the retinoblastoma protein family pRb (including p107). Once the E7N is fused with an E3 ligase, this E7N-E3 
complex could mediate the degradation of the whole family of $\mathrm{pRb}$ proteins in both mammalian and yeast systems [16]. In a follow-up study, this system was further improved to selectively degrade only p107 or the hypo-phosphorylated forms of retinoblastoma protein, respectively [17].

While these E3 engineering strategies mentioned above have led to rapid and selective POI degradation within cells, it cannot be easily extended to POIs without a naturally interacting protein partner. A more contemporary approach to TPD leverages the target protein recognition capacity of antibody-like fragments for intracellular protein targeting. A recent example of antibody linked E3 ligase is the development of small, stable, high-affinity variable region of a camelid heavy chain-only domain $\left(\mathrm{V}_{\mathrm{H}} \mathrm{H}\right)$ with specific binding to Botulinum neurotoxin (BoNT). When fused to an F-box domain region of TrCP D5 (Transducin Repeat Containing Protein D5), the $\mathrm{V}_{\mathrm{H}} \mathrm{H}-\mathrm{D} 5$ fusion mediates increased ubiquitination of intracellular BoNT and can accelerate the turnover of the targeted BoNT type A protease within neurons [18]. Because the $\mathrm{V}_{\mathrm{H}} \mathrm{H}$ anbibodies can be developed rapidly for nearly any in vivo protein targets, similar $\mathrm{V}_{\mathrm{H}} \mathrm{H}$ engineered TPD can be applied broadly to control the fast turnover of many target proteins. A further development of large-scale antibody-mediated TPD has been carried out in Drosophila melanogaster (fruit fly), which benefits from a library of transgenic flies with GFP tagged to almost every protein in its genome $\left[1^{\bullet \bullet}\right]$. Caussinus et al. cleverly fused a single-domain antibody fragment against GFP $\left(\mathrm{V}_{\mathrm{H}} \mathrm{HGFP}\right)$ to an F-box protein, which belongs to the E3 ligase complex, Slmb from Drosophila, to mediate the degradation of every GFP-fusion protein in the library $\left[20^{\bullet}\right]$. As opposed to the E3 ligase engineering using naturally interacting protein partners, antibody fused E3 ligases can, in theory, be easily adapted to target the whole proteome in any living organism. To further realize this hypothesis, Portnoff et al replaced the natural substrate 
binding domain of carboxyl-terminus of Hsc70-interacting protein (CHIP) with $\mathrm{V}_{\mathrm{H}} \mathrm{H}$ like antibodies to initiate the ubiquitination of various targets [21]. The modular CHIP E3 ligase was chosen to avoid the involvement of complex binding partners required for other types of E3 ligases, while striving to apply the antibody fused E3 ligase TPD strategy to virtually any endogenous protein in the cell (Figure 2A).

\section{Ubiquitination-independent pathway for TPD}

Uniquitination independent protein degradation of any protein can be achieved by simply fusing the C-degron to the C-terminus of a POI. Since the C-degron can result in the instability of a POI, this strategy usually precludes precise spatiotemporal control of protein abundance. To counter the protein stability issue, Renicke et al has leveraged a light responsive domain to control the protein stability by masking and exposing the fused C-degron [22]. The LOV (Light, Oxygen or Voltage) domain of the blue-light photoreceptor phototropin can respond to blue-light by conformation changes that will cause dissociation and unfolding of the J $\alpha$ helix extension at the C-terminus $[23,24]$. This system included a 23 amino acid long C-degron fused to the C-terminus of the LOV domain (Figure 2B). As long as this degron is masked by the Ja helix, the whole fusion protein is stable. Upon blue-light treatment, the unfolding of J $\alpha$ helix uncovers the Cdegron, which leads to ubiquitin-independent protein degradation. In addition, since the ODC degron based degradation mechanism is conserved in yeasts, vertebrates and plants, it can be applied to different organisms. Thus, light can serve as a general tool to precisely modulate protein abundance in vivo.

\section{Combining both ubiquitination dependent and independent pathways for TPD}


In terms of achieving TPD, ubiquitination dependent and independent protein degradation pathways can be used independently and in combination for a particular system. An excellent example of using this approach was demonstrated by using the tobacco etches virus (TEV) protease for mediating degron exposure. The tobacco etch virus (TEV) protease can recognize the canonical amino-acid sequence ENLYFQ-S/G and cleave at the position denoted by "-." $[25,26]$, leaving a sequence starting with S or G. S or G can be substituted by other residues with the cost of reduced recognition efficiency and lowered product stability [27].

One group has successfully developed a TEV protease TPD system by placing the POI after a TEV protease recognition sequence and the $\mathrm{S} /$ Gposition is substituted with an $\mathrm{F}$ or $\mathrm{D}[28,29]$. As such, the F/D in the cleavage product serves as an N-end degron to trigger the degradation of the POI [28]. An upgrade of this system introduced a C-degron before the cleavage site (Figure 2C), so that the protein fused before C-degron will also be degraded. In this way, the fusion proteins before and/or after the cleavage site can be either or both be degraded depending on the presence of C- and/or N-terminal degrons [29]. This TEV induced TPD can be easily adapted to control abundance of at least two proteins concurrently.

The aforementioned ubiquitination dependent and independent protein degradation pathways depend on the expression of an engineered protein as trigger signal (Figures 2A-2C). However, methodologies in which TPD trigger signal does not depend on the expression of a particular protein exist and will be discussed next.

\section{Plant hormones as novel inducing signals for TPD}

Leveraging plant hormones as triggering signals in target protein degradation represents an emerging trend. The auxin receptor TIR1 (Transport Inhibitor Response1) is an F-box E3 ligase 
that interacts and degrades members of the AUX/IAA (Auxin/Indole-3-Acetic Acid) protein family upon auxin perception. Auxin perception by TIR1 was found to strengthen the affinity between TIR1 and AUX/IAA [30]. An auxin-based TPD system has been developed in Saccharomyces cerevisiae and in vitro mammalian cell culture, where the TIR1 and the Targetprotein fused to IAA17 were expressed simultaneously and the IAA17 fused target protein will start to degrade after 30 min of auxin treatment [31].

A similar approach was also explored for jasmonates. Jasmonates are comprised of jasmonic acid (JA) and its derivatives, and jasmonate treatment invokes the interaction between three components: the F-box E3 ligase Coronatine-Insensitive1 (COI1), Jasmonate ZIM-domain (JAZ) and inositol pentakisphosphate [32]. Physical contact between COI1 and JAZ initiates the JAZ ubiquitination process upon jasmonate treatment [33,34]. Given that the virulence factor coronatine from plant pathogenic strains of Pseudomonas syringae causes much stronger JA response phenotypes [35], a coronatine and inositol pentakisphosphate inducible TPD system based on the jasmonate signaling pathway is currently under development [15].

These examples of utilizing plant hormones as novel TPD signals demonstrate that studying and mimicking the naturally occuring small molecules that induce protein degradation can be beneficial to developing high-efficient TPD systems (Figure 2D).

\section{Applications of TPD in cancer therapy}

Although various TPD strategies have been developed over the years to precisely modulate and monitor cellular functions, researchers are only beginning to adapt these post-translational tools for specific biomedical and biotechnology applications. Targeted Protein Degradation has 
recently been implemented as a novel tool in targeted cancer therapies [36,37]. In particular, Ma et al. determined that the degradation of the V-Ki-ras2 Kirsten rat sarcoma (KRAS) protein can be achieved by fusing its tight binding partner Ras-binding domains/Ras-associating domains (RBD/CRD) complex with a U-box E3 ligase. Lowered KRAS protein levels can suppress pancreatic cancer cell growth in vitro and in vivo [36]. Similarly, Zhong et al. leveraged the Epidermal growth factor receptor (EGFR) binding domain Sh2 fused to an ubiquitin ligase catalytic domain, such as CHIP, to demonstrate that the degradation of EGFR can inhibit malignancy of lung cancer SPC-A1 cells and sensitize these cells to chemotherapy [37]. Both examples indicate that TPD exhibits significant potential to be applied for targeted cancer therapy.

\section{Application of TPD for intracellular imaging of cell functions}

By fusing a fluorescence protein with C-degron, normal cells do not accumulate the fluorescence signal. However, cells with malfunctioned 26S proteasome can indeed accumulate trackable fluorescence signals, such as cancer initiating cells (cancer stem cell) or cells treated with radiation or chemotherapy. The fluorescence accumulation is thus used as a lineage tracker for a cancer initiating cell or as an activity indicator of 26S proteasome [38,39].

Fluorescence markers fused with degrons have been further developed to monitor the cell cycle transition in living cells. In a method named FUCCI (fluorescent ubiquitination based cell cycle indicator), green fluorescent protein is fused to Geminin (aa 1-110) and red fluorescent protein is fused to Cdt1 (aa 30-120) [40••]. Both fragments from Geminin and Cdt are degron signals.

Specifically, Geminin is degraded from mid-mitosis through G1 phase and Cdt is degraded in S 
and G2 phases. Thus, cells expressing both fluorescence proteins fused with degron display red in G1 phase and green in S, G2, and M phases.

A recent development of the degron based in vivo imaging technique is to build a degronprotease-blockade histone methylation sensor [41]. Sekar et al fused a luciferase to a H3-K9 peptide, and linked this fluorescent marker to the Suv39h1-chromodomain with C-degron at its end. The methylation process of the H3-K9 peptide by the methyltransferase will cause a conformation change in the Suv39h1-chromodomain to create a conformational lock of the Cdegron to prevent its degradation initiation. Thus, functional luciferase will accumulate in the cell to serve as an indicator for $\mathrm{H} 3-\mathrm{K} 9$ methylation status. The sensitivity of this $\mathrm{H} 3-\mathrm{K} 9$ methylation sensor has been verified in multiple cell lines as well as in animal models.

\section{Conclusions}

The conceptual framework and molecular basis of both ubiquitination dependent and independentTPD methods based on the expanded degron concept has expanded rapidly in recent years.

Compared with genome editing and RNA interference (RNAi) methodology, TPD offers a second and complimentary layer of phenotype engineering at the posttranslational level. Protein abundance control by TPD is more rapid than gene or mRNA disruption. In addition, TPD offers the possibility to remove residual long lived proteins, which may not be completely removed by RNAi methods. Indeed, combining RNAi and TPD methods may offer superior protein ablation efficiency [42].

Taken together, the multi-target functionality and ubiquitous nature of degron-based TPD in living organisms has wide ranging utility in cancer therapy, targeted protein toxin degradation, 
and intracellular process monitoring. With further development of $\mathrm{V}_{\mathrm{H}} \mathrm{Hs}$ as high-affinity antibodies within cells, conformation controllable proteins, and more permeable small molecules, degron based TPD will pave the way for proteome engineering in order to control multiple cellular activities and functions in the coming decades.

Acknowledgement

We thank the funding support from Emerging Frontiers in Research and Innovation program in National Science Foundation (Grant number: 1332344) 


\section{Figures}

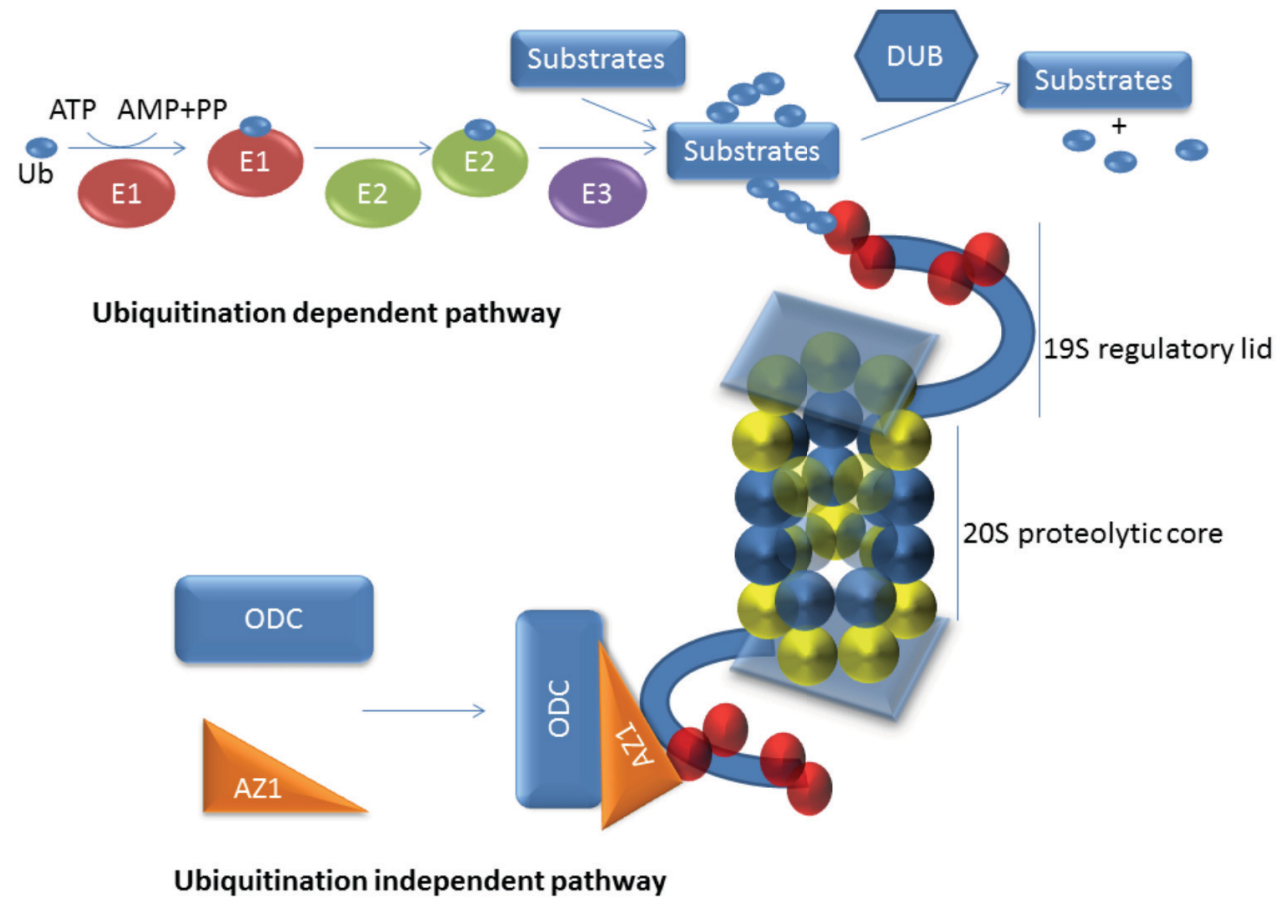

Figure 1. Schematic representation of degron-mediated protein degradation through proteasome. Proteasome is composed of a $20 \mathrm{~S}$ proteolytic core and two $19 \mathrm{~S}$ regulatory lids capping the core. The core contains four stacked $\alpha-\beta-\beta-\alpha$ rings. $\alpha$ heptamer ring is in yellow, $\beta$ heptamer ring is in blue. The 19S regulatory lid consists of a base with ATPase activity (the blue rectangular) and a scaffold structure with various receptors (red balls) attached. Ubiquitindependent pathway: ubiquitin $(\mathrm{Ub})$ monomer is activated by consuming an ATP and then transferred through a serious E1-E2-E3 enzymes onto specific residues of target substrates to form ubiquitin recognition signal, such as a poly-ubiquitin chain. Certain ubiquitin signal can be recognized by the receptors (red balls) on the proteasome and this signal can be further modified by DUB. Uniquitin-independent pathway: ODC is degraded by AZ1 mediated delivery to the proteasome. 


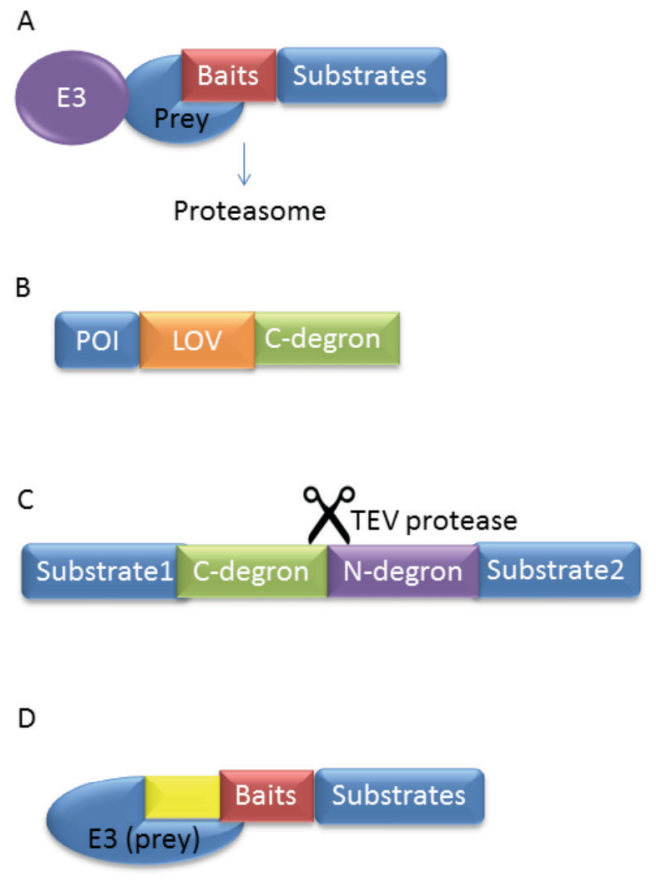

Figure 2. Schematic drawing of strategies used for TPD. A. Substrates can be brought to the proximity of $\mathrm{E} 3$ for ubiquination by utilization of interacting Baits-Prey pair, like E7N and $\mathrm{pRb}$ or antibody and antigen pair. B. The LOV domain linked C-degron can be linked to many POI for light inducible targeted protein degradation. C. C- and/or N-degrons can be built in between two unrelated substrates, which will be revealed after TEV protease cleavage. Depends on the presence of either or both $\mathrm{C}$-/N-degrons, substrates 1 and 2 can be either or both degraded. D. In plant hormone based TPD, the hormone receptor usually has both a E3 ligase and "prey" activity, but the "prey" can only interact with baits with the presence of specific plant hormone (yellow). 


\section{References}

Papers of particular interest, published within the period of review, have been highlighted as:

- of special interest

•• of outstanding interest

-1. Inobe T, Matouschek A: Paradigms of protein degradation by the proteasome. Current Opinion in Structural Biology 2014, 24:156-164.

This excellent review has provided a detailed mechanistic overview of protein degradation based onthe recently solved structure of proteasome.

2. Varshavsky A: Naming a targeting signal. Cell 1991, 64:13-15.

3. Varshavsky A: The $\mathbf{N}$-end rule pathway and regulation by proteolysis. Protein Science : A Publication of the Protein Society 2011, 20:1298-1345.

4. Fishbain S, Prakash S, Herrig A, Elsasser S, Matouschek A: Rad23 escapes degradation because it lacks a proteasome initiation region. Nat Commun 2011, 2:192.

5. Heinen C, Acs K, Hoogstraten D, Dantuma NP: C-terminal UBA domains protect ubiquitin receptors by preventing initiation of protein degradation. Nat Commun 2011, 2:191.

6. Prakash S, Inobe T, Hatch AJ, Matouschek A: Substrate selection by the proteasome during degradation of protein complexes. Nature chemical biology 2009, 5:29-36.

7. Baker TA, Sauer RT: ATP-dependent proteases of bacteria: recognition logic and operating principles. Trends in biochemical sciences 2006, 31:647-653.

8. Baugh JM, Viktorova EG, Pilipenko EV: Proteasomes Can Degrade a Significant Proportion of Cellular Proteins Independent of Ubiquitination. Journal of molecular biology 2009, 386:814827.

9. Finley D: Recognition and Processing of Ubiquitin-Protein Conjugates by the Proteasome. Annual review of biochemistry 2009, 78:477-513.

10. Tomko RJ, Hochstrasser M: Molecular Architecture and Assembly of the Eukaryotic Proteasome. Annual review of biochemistry 2013, 82:10.1146/annurev-biochem-060410-150257.

11. Lee B-H, Lee MJ, Park S, Oh D-C, Elsasser S, Chen P-C, Gartner C, Dimova N, Hanna J, Gygi SP, et al.: Enhancement of Proteasome Activity by a Small-Molecule Inhibitor of Usp14. Nature 2010, 467:179-184.

12. Bhattacharyya S, Yu H, Mim C, Matouschek A: Regulated protein turnover: snapshots of the proteasome in action. Nat Rev Mol Cell Biol 2014, 15:122-133.

13. Erales J, Coffino P: Ubiquitin-independent proteasomal degradation. Biochimica et Biophysica Acta (BBA) - Molecular Cell Research 2014, 1843:216-221.

14. Raina K, Crews CM: Chemical inducers of targeted protein degradation. J Biol Chem 2010, 285:11057-11060.

15. Faden F, Mielke S, Lange D, Dissmeyer N: Generic tools for conditionally altering protein abundance and phenotypes on demand. Biol Chem 2014, 395:737-762.

16. Zhou P, Bogacki R, McReynolds L, Howley PM: Harnessing the ubiquitination machinery to target the degradation of specific cellular proteins. Mol Cell 2000, 6:751-756. 
17. Zhang J, Zheng N, Zhou P: Exploring the functional complexity of cellular proteins by protein knockout. Proc Natl Acad Sci U S A 2003, 100:14127-14132.

18. Kuo CL, Oyler GA, Shoemaker CB: Accelerated neuronal cell recovery from Botulinum neurotoxin intoxication by targeted ubiquitination. PLoS One 2011, 6:e20352.

-19. Kelso RJ, Buszczak M, Quinones AT, Castiblanco C, Mazzalupo S, Cooley L: Flytrap, a database documenting a GFP protein-trap insertion screen in Drosophila melanogaster. Nucleic Acids Res 2004, 32:D418-420.

By expressing an anti-GFP antibody in Drosophila melanogaster, any protein been tagged by a GFP in the fly database could be subjected to TPD.

••20. Caussinus E, Kanca O, Affolter M: Fluorescent fusion protein knockout mediated by anti-GFP nanobody. Nat Struct Mol Biol 2012, 19:117-121.

By chosing a stand-alone E3 ligase CHIP, less complex protein degradation partners are required, thus expanding the range of the target protein selection.

21. Portnoff AD, Stephens EA, Varner JD, DeLisa MP: Ubiquibodies, synthetic E3 ubiquitin ligases endowed with unnatural substrate specificity for targeted protein silencing. $J$ Biol Chem 2014, 289:7844-7855.

22. Renicke C, Schuster D, Usherenko S, Essen LO, Taxis C: A LOV2 domain-based optogenetic tool to control protein degradation and cellular function. Chem Biol 2013, 20:619-626.

23. Christie JM, Salomon M, Nozue K, Wada M, Briggs WR: LOV (light, oxygen, or voltage) domains of the blue-light photoreceptor phototropin (nph1): binding sites for the chromophore flavin mononucleotide. Proc Natl Acad Sci U S A 1999, 96:8779-8783.

24. Harper SM, Neil LC, Gardner KH: Structural Basis of a Phototropin Light Switch. Science 2003, 301:1541-1544.

25. Carrington JC, Dougherty WG: A viral cleavage site cassette: identification of amino acid sequences required for tobacco etch virus polyprotein processing. Proc Natl Acad Sci US A 1988, 85:3391-3395.

26. Boulware KT, Jabaiah A, Daugherty PS: Evolutionary optimization of peptide substrates for proteases that exhibit rapid hydrolysis kinetics. Biotechnol Bioeng 2010, 106:339-346.

27. Kapust RB, Tozser J, Copeland TD, Waugh DS: The P1' specificity of tobacco etch virus protease. Biochem Biophys Res Commun 2002, 294:949-955.

28. Taxis C, Stier G, Spadaccini R, Knop M: Efficient protein depletion by genetically controlled deprotection of a dormant N-degron. Mol Syst Biol 2009, 5:267.

29. Jungbluth M, Renicke C, Taxis C: Targeted protein depletion in Saccharomyces cerevisiae by activation of a bidirectional degron. BMC Syst Biol 2010, 4:176-176.

-30. Tan X, Calderon-Villalobos LIA, Sharon M, Zheng C, Robinson CV, Estelle M, Zheng N: Mechanism of auxin perception by the TIR1 ubiquitin ligase. Nature 2007, 446:640-645.

This study provided an striking example of utilizing plant hormone as protein degradation controling mechanism.

31. Nishimura K, Fukagawa T, Takisawa H, Kakimoto T, Kanemaki M: An auxin-based degron system for the rapid depletion of proteins in nonplant cells. Nat Methods 2009, 6:917-922.

32. Sheard LB, Tan X, Mao H, Withers J, Ben-Nissan G, Hinds TR, Kobayashi Y, Hsu F-F, Sharon M, Browse J, et al.: Jasmonate perception by inositol phosphate-potentiated COI1-JAZ coreceptor. Nature 2010, 468:400-405. 
33. Chini A, Fonseca S, Fernandez G, Adie B, Chico JM, Lorenzo O, Garcia-Casado G, Lopez-Vidriero I, Lozano FM, Ponce MR, et al.: The JAZ family of repressors is the missing link in jasmonate signalling. Nature 2007, 448:666-671.

34. Thines B, Katsir L, Melotto M, Niu Y, Mandaokar A, Liu G, Nomura K, He SY, Howe GA, Browse J: JAZ repressor proteins are targets of the SCF(COI1) complex during jasmonate signalling. Nature 2007, 448:661-665.

35. Katsir L, Schilmiller AL, Staswick PE, He SY, Howe GA: COI1 is a critical component of a receptor for jasmonate and the bacterial virulence factor coronatine. Proceedings of the National Academy of Sciences 2008, 105:7100-7105.

36. Ma Y, Gu Y, Zhang Q, Han Y, Yu S, Lu Z, Chen J: Targeted degradation of KRAS by an engineered ubiquitin ligase suppresses pancreatic cancer cell growth in vitro and in vivo. Mol Cancer Ther 2013, 12:286-294.

37. Zhong D, Ru Y, Wang Q, Zhang J, Zhang J, Wei J, Wu J, Yao L, Li X, Li X: Chimeric ubiquitin ligases inhibit non-small cell lung cancer via negative modulation of EGFR signaling. Cancer Letters 2015.

38. Vlashi E, Kim K, Lagadec C, Donna LD, McDonald JT, Eghbali M, Sayre JW, Stefani E, McBride W, Pajonk F: In Vivo Imaging, Tracking, and Targeting of Cancer Stem Cells. Journal of the National Cancer Institute 2009, 101:350-359.

39. Brush JM, Kim K, Sayre JW, McBride WH, Iwamoto KS: Imaging of radiation effects on cellular 26S proteasome function in situ. Int J Radiat Biol 2009, 85:483-494.

• 40. Sakaue-Sawano A, Kurokawa H, Morimura T, Hanyu A, Hama H, Osawa H, Kashiwagi S, Fukami $\mathrm{K}$, Miyata $\mathrm{T}$, Miyoshi $\mathrm{H}$, et al.: Visualizing spatiotemporal dynamics of multicellular cellcycle progression. Cell 2008, 132:487-498.

By coupling the TPD and the methylation status inside cell, this study has further exemplied the broad application of degrons.

41. Sekar TV, Foygel K, Devulapally R, Paulmurugan R: Degron Protease Blockade Sensor to Image Epigenetic Histone Protein Methylation in Cells and Living Animals. ACS Chemical Biology 2015, 10:165-174.

42. Hannah J, Zhou P: Maximizing target protein ablation by integration of RNAi and protein knockout. Cell Res 2011, 21:1152-1154. 
Graphical Abstract

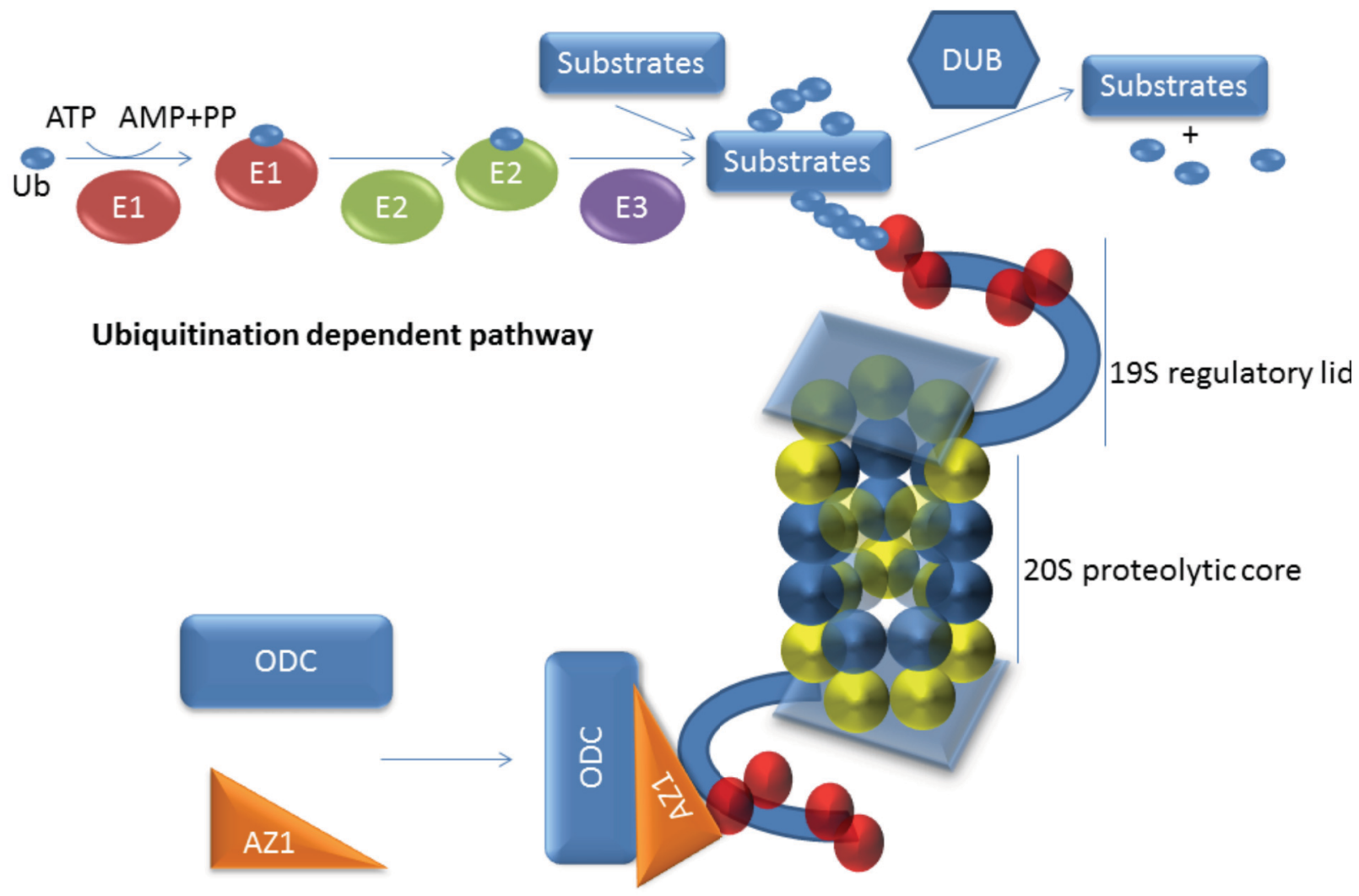

Ubiquitination independent pathway 